\title{
A Multi-Mode Viscosity Model and Its Applicability to Non-Newtonian Fluids
}

\author{
YASUDA Kazunori \\ Department of Mechanical Engineering, Graduate School of Engineering, Osaka University, \\ 2-1 Yamadaoka, Suita, Osaka 565-0871, Japan
}

Received 18 May 2006; accepted for publication 3 July 2006

\begin{abstract}
There are many viscosity equations to describe a shear-thinning viscosity of non-Newtonian fluid. In the present study, a multi-mode viscosity equation to more accurately fit measured data was proposed. The viscosity data were obtained for aqueous solutions of carboxymethylcellulose using a cone-and-plate rheometer. Consequently, the fitted results using the proposed viscosity equation to the measured data were very good.
\end{abstract}

Key Words: Non-Newtonian viscosity, Shear-thinning viscosity, Carreau equation, Multi-mode viscosity equation, CMC solution

\section{Introduction}

Shear viscosity is an important rheological property of a fluid. It is constant and independent of shear rate for a Newtonian fluid, while it depends on shear rate for a nonNewtonian fluid. The shear viscosity of a polymer solution, which is a typical non-Newtonian fluid, often decreases with an increase in shear rate. Such a fluid is called to have a shear-thinning viscosity. In general, even the shear-thinning fluid has a constant viscosity, which is independent of shear rate in a low and a high shear rate regions. These viscosities are called the zero-shear-rate viscosity and the infiniteshear-rate viscosity, respectively. A power-law region is also observed between these regions; when the viscosity is plotted as a function of shear rate in a log-log scale, the viscosity curve becomes a straight line.

Many viscosity equations as a function of shear rate are used to represent the shear-thinning viscosity [1-5]. In these models, Carreau equation [1] is well known as the most useful one, because it represents the zero-shear-viscosity, the power-law viscosity and the infinite-shear-rate viscosity very well. This model is reduced to Sisko model [2] and power-law model [3], but both the models have an infinite zero-shear-rate viscosity, which is unrealistic one. However, these models are often used because the model equations are very simple. Furthermore, the expanded Carreau model, which is called Carreau-Yasuda model [4], is widely used.
In the present paper, we will propose a newly modified Carreau model to describe the shear-thinning viscosity and compare the computed result using our model with the measured viscosity for polymer solutions.

\section{Viscosity model}

To predict the characteristic of fluid viscosity, many viscosity models have been proposed. The Cross model [5] is given by

$$
\frac{\eta-\eta_{\infty}}{\eta_{0}-\eta_{\infty}}=\frac{1}{1+(\lambda \dot{\gamma})^{m}}
$$

in which $\eta$ is the shear viscosity, $\eta_{0}$ the zero-shear-rate viscosity, $\eta_{\infty}$ the infinite-shear-rate viscosity, $\lambda$ a parameter with unit of time, $\dot{\gamma}$ shear rate and $m$ a dimensionless parameter. This model is reduced to the power-law model for $\eta<<\eta_{0}$ and $\eta>\eta_{\infty}$

$$
\eta=K \dot{\gamma}^{n-1},
$$

where $K$ and $n$ are called the "consistency" and the powerlaw index, respectively. Furthermore, when $\eta \ll \eta_{0}$, we obtain

$$
\eta=\eta_{\infty}+K \dot{\gamma}^{n-1} .
$$

This is called the Sisko model [2].

The alternative to the Cross model is the four-parameter

* Corresponding author: E-mail: yasuda@ mech.eng.osaka-u.ac.jp, Tel/Fax: +81-6-6879-7309 
Carreau model [1] which is widely used and it is given by the following equation:

$$
\frac{\eta-\eta_{\infty}}{\eta_{0}-\eta_{\infty}}=\left\{1+(\lambda \dot{\gamma})^{2}\right\}^{(n-1) / 2}
$$

In the above models, it is difficult to measure the infiniteshear-rate viscosity $\eta_{\infty}$, so it is often set to be very small value. Thus, for the aqueous solution used in the present measurements, $\eta_{\infty}$ was set to be 0 Pa.s $(\eta \approx 1.0 \mathrm{mPa} . \mathrm{s})$. Then, the Carreau model is reduced to the following equation:

$$
\eta=\eta_{0}\left\{1+(\lambda \dot{\gamma})^{2}\right\}^{(n-1) / 2} .
$$

Yasuda et al. [4] modified the Carreau model and obtained the Carreau-Yasuda model:

$$
\eta=\eta_{0}\left\{1+(\lambda \dot{\gamma})^{a}\right\}^{(n-1) / a},
$$

where $a$ is a parameter to describe the transition region between the zero-shear-rate and power-law regions.

\section{Test materials}

We prepared three aqueous solutions of carboxymethylcellulose (CMC, 2260, Daicel Chemical Industries, LTD., Japan) as test fluids. The concentrations are $0.5 \mathrm{wt} \%, 1 \mathrm{wt} \%$ and $2 \mathrm{wt} \%$. The CMC was added to distilled water and fully stirred over one night. The shear viscosities of the test fluids were measured using a cone-and-plate rheometer (Physica MCR300) with $1^{\circ}$ in cone angle and $50 \mathrm{~mm}$ in diameter. Our measurements were carried out at temperature of $25^{\circ} \mathrm{C}$.

\section{Results and discussion}

The measured results were plotted in terms of the shear viscosity and the shear rate in Fig. 1. We see from this

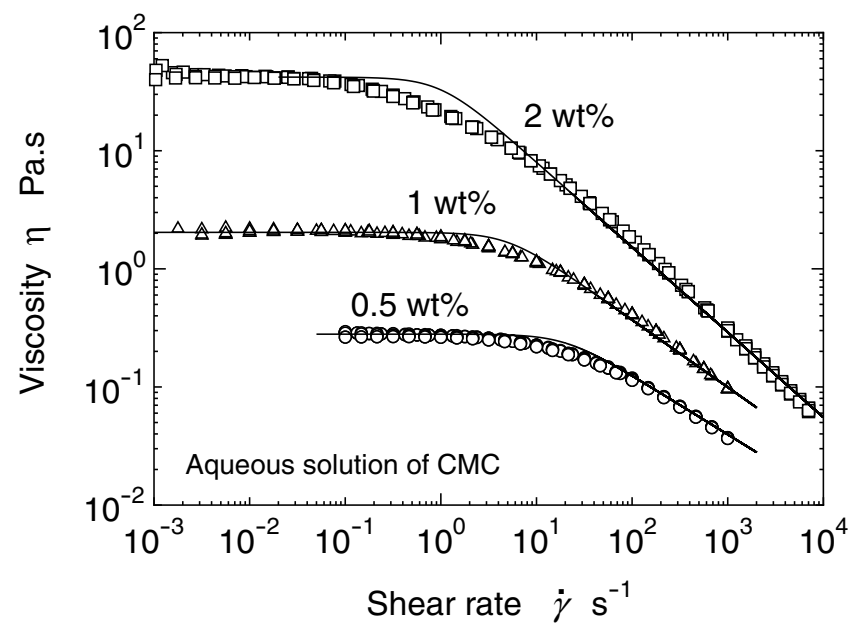

Fig. 1 Shear viscosities of aqueous solutions of carboxymethylcellulose at temperature of $25^{\circ} \mathrm{C}$. The solid curves represent the Carreau equations for the $0.5 \mathrm{wt} \%, 1 \mathrm{wt} \%$ and $2 \mathrm{wt} \% \mathrm{CMC}$ aqueous solutions.
Table 1 Parameters of the Carreau model for the test fluids.

\begin{tabular}{l|lll}
\hline \hline & \multicolumn{3}{|c}{ CMC } \\
\cline { 2 - 4 } & $0.5 \mathrm{wt} \%$ & $1 \mathrm{wt} \%$ & $2 \mathrm{wt} \%$ \\
\hline$\eta_{0}$ (Pa.s) & 0.27 & 2.2 & 42 \\
$\lambda(\mathrm{s})$ & 0.05 & 0.2 & 1 \\
$n(-)$ & 0.50 & 0.43 & 0.28 \\
\hline
\end{tabular}

figure that CMC solution has a typical non-Newtonian viscosity: zero-shear-rate viscosity and shear-thinning one. The results of fitting these data to the Carreau equation (solid curves) are shown in the same figure. The parameters of the Carreau equation for these test fluids are summarized in Table 1. From this figure we see that the fitted curves obtained using the Carreau equation are not in good agreement with the measured data except in the low and high shear rate regions. The difference between the fitted result and the measured data becomes large with an increase in concentration of CMC. The shear viscosity expressed by the Carreau model decreases over the shear rate of $\lambda \dot{\gamma}=1$, and we cannot adjust the breadth of the transition region between the zero-shear-rate viscosity and the power-law region.

In the present paper, we have proposed the following multi-mode viscosity equation for the fitted curves to be in good agreement with the measured data instead of eqn. (5):

$$
\eta=\eta_{0} \prod_{i=1}^{k}\left\{1+\left(\lambda_{i} \dot{\gamma}\right)^{2}\right\}^{\left(n_{i}-1\right) / 2} .
$$

This model has a lot of parameters $\lambda_{i}$ with unit of time and $n_{i}$ which is dimensionless $(i=1,2,3 \ldots)$. Here, we can regard these parameters $\lambda_{i}$ as relaxation times having widely distributing values.

Figure 2 shows the fitted results (solid curves) using the

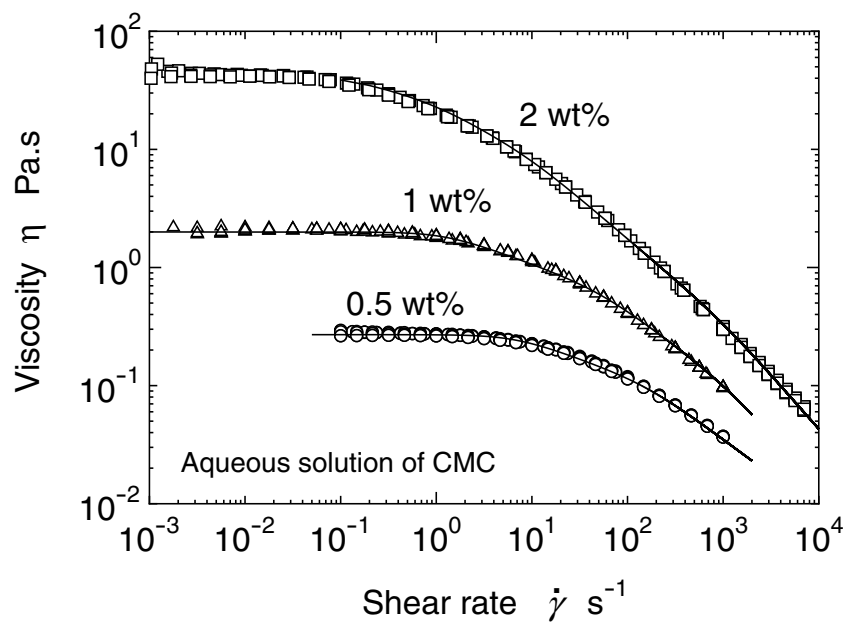

Fig. 2 Shear viscosities of aqueous solutions of carboxymethylcellulose at temperature of $25^{\circ} \mathrm{C}$. The solid curves represent the multi-mode viscosity equations for the $0.5 \mathrm{wt} \%, 1 \mathrm{wt} \%$ and $2 \mathrm{wt} \% \mathrm{CMC}$ aqueous solutions. 
Table 2 Parameters of the multi-mode viscosity model for the test fluids.

\begin{tabular}{l|lll}
\hline \hline & \multicolumn{3}{|c}{ CMC solution } \\
\cline { 2 - 4 } & $0.5 \mathrm{wt} \%$ & $1 \mathrm{wt} \%$ & $2 \mathrm{wt} \%$ \\
\hline$\eta_{0}$ (Pa.s) & 0.27 & 2.2 & 42 \\
$\lambda_{1}(\mathrm{~s})$ & 0.15 & 0.93 & 10 \\
$\lambda_{2}(\mathrm{~s})$ & 0.006 & 0.1 & 1 \\
$\lambda_{3}(\mathrm{~s})$ & - & 0.017 & 0.1 \\
$\lambda_{4}(\mathrm{~s})$ & - & - & 0.03 \\
$n_{1}(-)$ & 0.70 & 0.70 & 0.70 \\
$n_{2}(-)$ & 0.73 & 0.90 & 0.88 \\
$n_{3}(-)$ & - & 0.80 & 0.80 \\
$n_{4}(-)$ & - & - & 0.86 \\
\hline
\end{tabular}

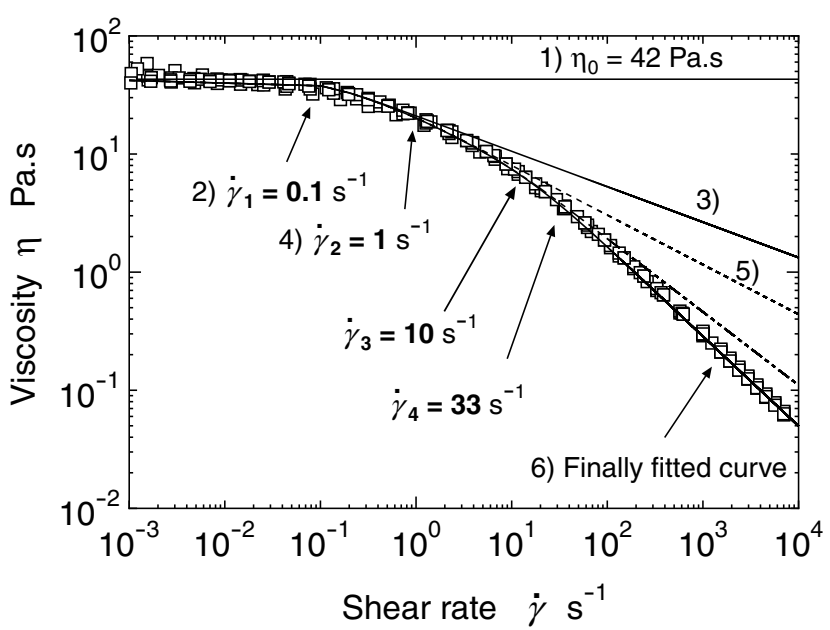

Fig.3 Determination of parameters of multi-mode viscosity model for the $2 \mathrm{wt} \% \mathrm{CMC}$ aqueous solution.

above viscosity model. The values of parameters are summarized in Table 2 . In our viscosity model, we can easily fit the model curves with the measured data even in the transition region using various values of parameters $\lambda_{i}$ and $n_{i}$. Let us determine these parameters through the following process. The process number 1), 2), 3) ... corresponds to the each one shown in Fig. 3.

1) Estimate the zero-shear-rate viscosity $\eta_{0}$ : it is easy to determine this parameter.

2) Estimate the shear rate $\dot{\gamma}_{1}$ at which the viscosity begins to decrease, then calculate $\lambda_{1}$ using the relation $\lambda_{1} \dot{\gamma}_{1}=1$.

3) Fit the model curve to the measured data in the region of shear rate higher than $\dot{\gamma}_{1}$ with varying the parameter $n_{1}$. Then, we can obtain the following equation:

$$
\eta=\eta_{0}\left\{1+\left(\lambda_{1} \dot{\gamma}\right)^{2}\right\}^{\left(n_{1}-1\right) / 2} .
$$

4) Estimate the shear rate $\dot{\gamma}_{2}$ at which the fitted curve 3) departs from the measured data of viscosity. We can calculate $\lambda_{2}$ using the relation $\lambda_{2} \dot{\gamma}_{2}=1$.

5) Fit the model curve to the measured data in the region of shear rate higher than $\dot{\gamma}_{2}$ with varying the parameter $n_{2}$. Then, we can obtain the following equation:

$$
\eta=\eta_{0}\left\{1+\left(\lambda_{1} \dot{\gamma}\right)^{2}\right\}^{\left(n_{1}-1\right) / 2}\left\{1+\left(\lambda_{2} \dot{\gamma}\right)^{2}\right\}^{\left(n_{2}-1\right) / 2} .
$$

6) When you cannot fit the model curve to the measured data accurately, repeat the further process to determine the parameter combinations $\left(\dot{\gamma}_{3}, n_{3}\right),\left(\dot{\gamma}_{4}, n_{4}\right) \ldots$. through the above process. Finally, we can obtain the eqn. (7).

We can find from this figure that the fitted curves are in very good agreement with the measured data. The CarreauYasuda model [4] shown in eqn. (6) is well known as the viscosity model which has good applicability to the shearthinning viscosity. The present multi-mode viscosity model is also very effective to represent the shear-thinning viscosity.

\section{Conclusion}

We proposed a modified viscosity equation to fit the measured viscosity data. The viscosity data were obtained for the aqueous solutions of carboxymethylcellulose using a cone-and-plate rheometer at temperature of $25^{\circ} \mathrm{C}$. Consequently, the predicted results of our equation were in good agreement with the measured data.

\section{References}

[1] Carreau PJ (1972) Trans Soc Rheol, 16, 99-127

[2] Sisko AW (1958) Ind Eng Chem, 50, 1789-1792

[3] Barnes HA, Hutton JF, Walters K (1989) "Introduction to Rheology", p19, Elsevier, Amsterdam

[4] Yasuda K, Armstrong RC, Cohen RE (1981) Rheol Acta, 20, $168-178$

[5] Cross MM (1965) J Colloid Sci, 20, 417-437 